\title{
Propiedades físicas y químicas de cultivares de cacao (Theobroma cacao L.) de Ecuador y Perú
}

\section{Physical and chemical properties of cacao cultivars (Theobroma cacao L.) from Ecuador and Peru}

\author{
Andrade-Almeida J', Rivera-García $J^{2}$, Chire-Fajardo GC ${ }^{2}$, Ureña-Peralta $M O^{2}$
}

\section{Resumen:}

El objetivo de este trabajo fue evaluar las propiedades físicas y químicas de los granos de cacao Nacional fino de aroma y CCN 51 procedentes de Ecuador, así como ICS 6 y CCN 51 originarios de Perú para la elaboración de chocolate de calidad. Se determinaron índice de grano (g), porcentaje de testa, dimensiones de grano (mm), porosidad (\%), índice de fermentación (\%), índice de blancura, acidez (\%), pH y análisis proximal (\%), y se utilizaron los métodos AOAC y los usuales en calidad del cacao. Referente al análisis proximal: el contenido de proteína cruda promedio del cacao peruano fue 78.8 \% superior; el contenido de grasa cruda promedio del cacao de Ecuador fue $10.1 \%$ superior; el contenido de fibra cruda del cacao ICS 6 peruano destaca entre los demás granos de cacao con $7.10 \pm 0.28 \%$. Los demás componentes tienen un grado de similitud entre ambos países, por lo que ambos son aptos para ser utilizados en la producción de chocolate de alta calidad, debido a sus atributos químicos y sensoriales, lo cual genera un producto de calidad y competitivo a escala internacional, reafirma el interés desde otras latitudes, impulsa el proceso de exportación y el beneficio económico para cada nación.

\section{Palabras clave}

Cultivar de cacao; caracterización; fino de aroma; CCN 51, ICS 6.

\begin{abstract}
The objective of this work was to evaluate the physical and chemical properties of the fine National flavor cocoa beans and CCN 51 from ECuador, as well as, ICS 6 and CCN 51 from Peru for the production of quality chocolate. They were determined, cacao bean index (g), cacao bean testa percentage, cacao bean dimensions (mm), porosity (\%), fermentation index (\%), whiteness index, acidity (\%), pH and proximate analysis (\%), using AOAC methods and the usual ones for cacao quality. According to proximate analysis: the average crude protein content for Peruvian cacao bean was $78.8 \%$ higher, the average crude fat content for Ecuador cacao bean was $10.1 \%$ higher, the crude fiber of ICS 6 cacao bean stands out among the other cocoa beans with $7.10 \pm 0.28 \%$, other components have a degree of similarity between the two countries, so both are suitable to be worked in the production of high quality chocolate, due to its chemical and sensory attributes, generating a quality and competitive product internationally, certifying interest from other latitudes drive the export process and economic benefit for each nation.
\end{abstract}

\section{Keywords}

Cultivate cocoa; characterization; fine aroma; CCN 51; ICS 6.

\section{Introducción}

A lo largo de la historia, el cacao ha sido un rubro de importancia económica y social en distintas culturas del continente americano, especialmente fue un producto simbólico dentro de la cultura incaica, en la que cumplió una función tanto para el consumo como para el comercio, ya que sirvió como una especie de moneda para realizar intercambios entre comunidades y grupos aborígenes. Por esta razón, en la actualidad se está sembrando variedades de especies,

$1 \quad$ Universidad Laica Eloy Alfaro de Manabí, Manabí-Ecuador (jacinto.andrade@uleam.edu.ec)

2 Universidad Nacional Agraria La Molina, Lima-Perú (johantheobroma@gmail.com; gchire@lamolina.edu.pe; moup@lamolina. edu.pe) 
como es el caso del cacao fino de aroma, que gracias a sus bondades químicas y sensoriales es exportado a distintas latitudes, donde tiene relevante aceptación.

En Suramérica existen varietales de cacao dentro de la región, donde resaltan países como Ecuador, Brasil, Perú, Colombia y Venezuela. Ecuador y Perú son reconocidos por el mejor grano y por el cacao fino de aroma (MINAGRI, 2016) en Ecuador el autor Castro-Zurita a través de sus investigaciones desde el año 1952 con diversas variedades de cacao, obtuvo un hibrido, que resalta por ser tolerante a las enfermedades, y que es de alta productividad y calidad, denominado clon CCN 51 que significa Colección Castro Naranjal \#51, este se creó en el año1965. Posteriormente el 22 de junio del 2005 fue declarado, mediante acuerdo ministerial, "un bien de alta productividad" con características diferentes al cacao Nacional (ANECACAO, 2019). En Ecuador también se tiene el cacao Nacional con características individuales distintas que lo hacen único y especial, el cual sobresale por su conocido sabor "arriba" (FAO-IICA, 2007).

En el Perú se cultiva el cacao ICS 6, que fue seleccionado dentro de los siete cultivares universales de origen trinitario, los cuales cumplen las características de finos de aroma (Alianza Cacao Perú, 2016). Sin embargo, estas características pueden verse afectadas por una inadecuada fermentación, pero con una correcta fermentación presenta los siguientes valores: 0-2 \% granos de cacao pizarrosos; granos de cacao parcial o totalmente violetas no mayores al $35 \%$; y granos de cacao marrones con $65 \%$, porque al exceder estos valores puede haber una sobrefermentación (Stevenson, Corven y Villanueva, 1993). Contrario a este criterio, se dice que la fermentación del cacao debe ser superior a un $75 \%$ con granos de cacao marrones, lo que indica un buen manejo en el proceso (Amores, Palacios, Jiménez y Zhang, 2009). Cabe resaltar, que este genotipo de cacao, CCN 51, se encuentra también en el Perú. Actualmente, la diferenciación de los granos con fines comerciales de estos dos tipos de cacao es un reto que ha sido investigado mediante distintas técnicas analíticas; por ello, se afirma que los granos fermentados de cacao son los ingredientes más importantes para la diversidad de productos en la industria chocolatera (Vargas, Ciobota, Salinas, Kampe, Aponte, et al, 2016).

En Ecuador y Perú se fermenta el grano de cacao para la producción de chocolate utilizando cajones fermentadores de diversos materiales, tales como: cajas de madera, plástico, y sacos de cabuya. En ese contexto, se toma como referencia en ambas naciones el ensayo realizado en Ecuador con cacao Nacional, del cual se obtuvo mayor porcentaje de granos de cacao fermentados en cajas de madera, comparados con los fermentados en las cajas plásticas; asimismo, se tiene menor porcentaje de granos de cacao violetas en cajas de madera y mayor porcentaje en cajas plásticas. Otra manera de fermentar el grano es por medio de la técnica del montón, conocida como montículos de granos de cacao colocados sobre una superficie abierta y cubiertos con hojas. De igual manera, se conoce la técnica aplicada con sacos de cabuya, que consiste en colocar los granos de cacao dentro del saco y dejarlos fermentar por un tiempo estimado según el tipo de cultivar (Rivera, Mecías, Guzmán, Peña, Medina, et al, 2012).

Cabe destacar que el proceso de diseño y elaboración del cajón fermentador tiene influencia en el índice de fermentación (IF), lo que afecta ligeramente en los niveles de fermentación en el caso del cajón cuadrado en comparación con el cajón fermentador rectangular (Nogales, Graziani y Ortiz, 2006). En este sentido, se toma la investigación de Ruiz, Mera, Prado y Cedeño (2015), para indicar que en el porcentaje de fermentación y generación de granos de cacao violetas son influenciados por época de cosecha, puesto que durante el período seco es mayor el porcentaje de fermentación y menor el número de granos de cacao violetas. 
Un estudio de relevancia fue elaborado por Álvarez, Tovar, García, Morillo, Sánchez, et al. (2010), en el cual se seleccionó de manera aleatoria 100 granos de cacao fermentados en cajas de madera (CM) y cajas plásticas (CP), como resultando se obtuvo un valor superior en CM $157.45 \mathrm{~g}$ y menor en CP $148.89 \mathrm{~g}$ y el porcentaje de testa fue mayor en CP 14.04 y en CM 13.95. Al respecto del estudio de Álvarez, se evidencia que la proporción de testa tiene relación con el peso del grano de cacao y es importante dentro de la industria de tostado del cacao, perdiendo porcentaje de grasa, migrando a la cáscara, lo que no es conveniente para la producción (Álvarez, Pérez y Lares, 2007).

Otro estudio de análisis del contenido de grasa se desarrolló en Colombia con 12 materiales de cacao con un rango de 51.4 a $57.7 \%$. En sus valores de fibra muestran un rango amplio 1.1 a $4.1 \%$, y el material de mayor valor de fibra fue el cacao SCC 52 con 4.1 \% (Perea, Ramírez y Villamizar, 2011).

Otras investigaciones reportan que las condiciones ambientales como la temperatura y la humedad afectan el contenido del grano de cacao (Chire, Verona y Guzmán, 2016), siendo la humedad un factor de mayor relevancia, ya que a valores mayores a $8 \%$ se produce el deterioro del grano por la presencia de microorganismos, especialmente de hongos. Asimismo, la humedad menor al $6 \%$ también es perjudicial, pues el grano puede volverse quebradizo (Aguilar, 2016). Mediante la prueba de Duncan al $5 \%$, se encontró diferencias significativas en lo reportado con mayores porcentajes de acidez, grasa, humedad y proteínas en los cacaos criollos, y los valores más altos de pH y taninos en los granos de cacao forasteros (Ortiz, Graziani y Rovedas, 2009). Por último, se diferencian genotipos evaluados por Pérez, Álvarez y Lares (2002) en una muestra comercial procedente de la región cacaotera venezolana de Chuao, con respecto al análisis de contenido de humedad, grasas y cenizas.

El objetivo de esta investigación fue describir las propiedades físicas y químicas de los cultivares de cacao Nacional fino de aroma y CCN 51 procedentes de Ecuador, así como de ICS 6 y CCN 51 originarios de Perú, con el propósito de identificar las características de los granos de cacao para la producción de chocolate de calidad.

\section{Metodología}

Se procedió a hacer las mediciones físicas a partir de técnicas e instrumentos propios del laboratorio y las químicas con métodos AOAC, método 942.15, entre otros. Con materiales de granos de cacao obtenidos de Ecuador, en la provincia de Manabí, el cacao Nacional con tres días de fermentación fue adquirido en la empresa Fortaleza del Valle ubicada en la ciudad de Calceta; mientras que el material CCN 51 con seis días de fermentación fue adquirido en los predios de un agricultor en el cantón Pedernales. A su vez, los granos de cacao procedentes de Perú, de la provincia de Tocache, región San Martín del Fundo Terra Nostra, ICS 6 con cinco días y CCN 51 seis días de fermentación, todo ello desde el análisis y proyección comercial.

El escenario de análisis fue desarrollado dentro del laboratorio de investigación de la Facultad de Industrias Alimentarias de la Universidad Nacional Agraria La Molina y en la Universidad Laica Eloy Alfaro de Manabí, Campus Pedernales.

En cuanto a las fases del trabajo, se inició con la cosecha de las mazorcas de los cultivares de ambos países en un estado de madurez óptimo. Los granos fueron extraídos el mismo día de la cosecha, fermentados adecuadamente y secados al sol. 
Las propiedades físicas comprenden las dimensiones del grano de cacao que fueron medidas con un verniere Stainless Hardened (R 2-10 mm (Chire, Orosco, Valdivia y Ureña, 2014). Para determinar el índice de grano (IG) (APPCACAO, 2012), se procedió a pesar 100 granos de cacao, dividido para 100, en una balanza Analítica Adventurer ${ }^{\circledR}$, con un rango de medición (10-4 g) Mod. AR 2140. Para el porcentaje de testa se pesó 30 granos de cacao, luego se retiró manualmente la testa y se reportó el porcentaje de cascarilla de la muestra respecto al índice de semilla (Vera, Vallejo, Párraga, Morales, Macías, et al, 2014).

La porosidad ( $\varepsilon$ ) se determinó conociendo la densidad real del grano de cacao, en un porosímetro de Day modificado por Ureña (1990), así como la densidad aparente del grano de cacao. Se pesa un total de 50 gramos de cacao y se insertan en una probeta de $250 \mathrm{~mL}$, y se relaciona masa (kg) y el volumen (L). Para calcular el índice de fermentación (IF), se procedió a cortar longitudinalmente 300 granos para observar el grado de fermentación, de acuerdo con la coloración presente en el interior de los granos de cacao. Se consideran granos de cacao bien fermentados a los que presentaron estrías bien pronunciadas y el color marrón o café rojizo. Los granos que tienen menos estrías y color púrpura son considerados granos de cacao medianamente fermentados, y los de color violeta o pizarroso, con ligeras estrías y compactas, se los consideró granos de cacao no fermentados, lo que permite, de esta manera, contabilizar los granos de cacao bien fermentados para calcular el índice de fermentación (INACAL, 2016; Gutiérrez, 2007).

El color se determinó por índice de blancura (IB). El IB significa el grado de blancura que tiene un alimento; a mayor valor de IB es más blanco o claro, y a menor valor de IB es al contrario (Lohman y Hartel, 1994). Para ello, se preparó la muestra vertiéndola en un molino de café con cuchillas trituradoras hasta que quedó en partículas muy finas, luego se cernió con un tamiz de $100 \mu \mathrm{m}$ y después se procedió a medir con el colorímetro digital (Konica Minolta CR4), para determinar los índices de blancura y conocer los valores de $L^{*}, a^{*} y b^{*}$ para obtener el IB mediante la aplicación de la siguiente fórmula:

$$
I B=100-\sqrt{\left(100-L^{*}\right)^{2}+a^{2}+b^{*^{2}}}
$$

Leyenda:

$I B=$ índice de blancura,

$L^{*}=$ luminosidad,

$a^{\star}=$ coordenadas rojo/verde, $y$

$b^{\star}=$ coordenadas amarillo/azul.

El análisis químico comprendió la medición de la acidez titulable, expresada como porcentaje de ácido acético, utilizando el método 942.15 (AOAC, 2016).

También se determinó el valor pH utilizando un potenciómetro SCHOTT, Handylab D-55014 Mainz®, método 970.21 (AOAC, 2016) y los que comprenden el proximal como la humedad 931.04, proteína cruda 970.22, grasa cruda 963.15, cenizas 972.15, fibra cruda 930.20a y 962.09 e, y los carbohidratos totales se determinaron restando del $100 \%$ el porcentaje de los compuestos ya determinados.

El proceso de análisis fue desarrollado con el programa Statgraphics Centurion XVIII, el cual permite ingresar los parámetros físicos y químicos para analizar la existencia estadística significativa menor al $5 \%$ de los objetos de estudio. 


\section{Resultados y Discusión}

Dimensiones del grano: Las medidas de dimensiones de los granos de cacao se muestran en la Tabla 1. El cacao Nacional de Ecuador tuvo los siguientes valores: largo $21.96 \pm 0.13 \mathrm{~mm}$, ancho $12.32 \pm 0.17 \mathrm{~mm}$ y espesor $8.19 \pm 0.09 \mathrm{~mm}$, que mostró una diferencia de medidas en el largo con el ICS 6 ( $p \leq 0.05$ ) de Perú; estas medidas fueron largo $23.39 \pm 1.24$ mm, ancho 12.82 $\pm 0.34 \mathrm{~mm}$ y espesor $9.32 \pm 1.38 \mathrm{~mm}$. El material CCN 51 de Ecuador mostró valores de largo $22.45 \pm 0.32 \mathrm{~mm}$, ancho $12.79 \pm 0.16 \mathrm{~mm}$, y espesor $8.24 \pm 0.36 \mathrm{~mm}$; frente al CCN 51 de Perú con valores de largo $24.97 \pm 0.40 \mathrm{~mm}$, ancho $13.80 \pm 0.89 \mathrm{~mm}$ y espesor $9.78 \pm 0.94 \mathrm{~mm}$, con diferencia significativa $(p \leq 0.05)$ en el largo y ancho, siendo el cacao CCN 51 de Perú el más largo y ancho de los granos de cacao estudiados para ambos países. Las dimensiones de los granos de cacao de Ecuador fueron menores y las dimensiones de los granos de cacao de Perú fueron similares a lo reportado en Colombia, donde se muestra resultados de: largo 24.5 a 24.8 mm, ancho 13.3 a 13.4 mm y espesor 9.5 a 10.7 mm (Álvarez et al., 2010). En un ensayo realizado en Chiapas (México), en varios cultivares de cacao los resultados de dimensiones no difieren mayormente de los resultados de este estudio (Caballero, Avendaño, González y López, 2016).

Tabla 1. Dimensiones de granos de cacao, en largo, ancho y espesor ( $\mathrm{mm}$ )

\begin{tabular}{|l|l|l|l|}
\hline \multirow{2}{*}{ Cultivares de cacao } & \multicolumn{3}{|c|}{ Variables } \\
\cline { 2 - 4 } & \multicolumn{1}{|c|}{${\text { Largo }(\mathrm{mm})^{*}}^{*}{\text { Ancho }(\mathrm{mm})^{*}}^{*}{\text { Espesor }(\mathrm{mm})^{*}}^{*}$} & $8.19 \pm 0.09^{\mathrm{a}}$ \\
\hline Nacional Ecuador & $21.96 \pm 0.13^{\mathrm{c}}$ & $12.32 \pm 0.17^{\mathrm{b}}$ & $8.24 \pm 0.36^{\mathrm{a}}$ \\
\hline CCN 51 Ecuador & $22.45 \pm 0.32^{\mathrm{bc}}$ & $12.79 \pm 0.16^{\mathrm{b}}$ & $9.78 \pm 0.94^{\mathrm{a}}$ \\
\hline CCN 51 Perú & $24.97 \pm 0.40^{\mathrm{a}}$ & $13.80 \pm 0.89^{\mathrm{a}}$ & $9.32 \pm 1.38^{\mathrm{a}}$ \\
\hline ICS 6 Perú & $23.39 \pm 1.24^{\mathrm{b}}$ & $12.82 \pm 0.34^{\mathrm{b}}$ & \multicolumn{2}{|c|}{} \\
\hline
\end{tabular}

*La desviación estándar seguida de letras distintas en la misma columna es significativamente diferente $(p<0.05)$.

Índice de grano (IG): En la Tabla 2, se muestra el índice de grano de los varietales de Ecuador y Perú, donde se indica que el cacao Nacional tuvo un valor de $1.24 \pm 0.03$ g y CCN 51 con un valor de $1.50 \pm 0.02 \mathrm{~g}$. Los materiales de Perú presentaron valores para ICS 6 de $1.30 \pm 0.01$ y para CCN 51 de $1.41 \pm 0.06$. Existiendo diferencia significativa ( $p \leq 0.05)$ entre todos los granos de cacao, estos valores fueron inferiores a los granos de cacao reportados en Ecuador (Vera et al., 2014). El CCN 51 de Ecuador tuvo el mayor valor de índice de grano, y el Nacional de Ecuador, el menor. En el tostado del cacao, los granos pequeños deberán tener una temperatura y tiempo de tostado distintos a los granos de mayor tamaño, de lo contrario se quemarán, por lo que es primordial conocer el índice de grano. Para el caso del cacao Nacional de Ecuador, se necesita menor temperatura y tiempo de tostado en comparación con los demás granos de cacao para tener un chocolate de calidad; posteriormente al tostado se pueden mezclar los granos de cacao para la producción de chocolate.

Porcentaje de testa: Los porcentajes de testa de los varietales de Ecuador muestran que el cacao Nacional tuvo un valor de $16.19 \pm 0.49 \%$ y CCN 51, un $12.33 \pm 0.34 \%$. Los materiales de Perú mostraron los siguientes resultados: CCN 51 con $10.05 \pm 0.54 \%$, y para ICS 6 con $15.38 \pm 0.37 \%$. El grano de cacao Nacional de Ecuador e ICS 6 de Perú no muestran diferencias significativas ( $p>0.05)$; sin embargo, el grano de cacao CCN 51 de ambos países muestran diferencias significativas $(p \leq 0.05)$. En el ensayo de la región venezolana de Cuyagua se obtuvieron 
resultados del porcentaje de testa que fueron desde 14.81 a $15.12 \%$, y se observa que a mayor tamaño de grano de cacao, presenta un mayor porcentaje de testa (Álvarez et al., 2007). En otras investigaciones, el porcentaje de testa de cacao comercial fue de 11.3, y de $15.5 \%$ en las regiones ecuatorianas de Lago Agrio y Santo Domingo respectivamente (Alegría, 2015). En los cultivares que fueron evaluados en tres regiones agroecológicas de Colombia, se obtuvieron resultados de porcentaje de testa o cascarilla de 10.05 hasta $16.22 \%$, y relacionando estos porcentajes de testa con el peso del grano, se determinó que es inversamente proporcional; es decir, que a mayor tamaño de grano menor porcentaje de testa, y a menor tamaño de grano mayor porcentaje de testa (FEDECACAO, 2004). Los valores de porcentaje de testa en los granos de cacao de los dos países están dentro de los valores mencionados, pero estos resultados son inversos al tamaño del grano, lo que coincide con lo reportado por FEDECACAO.

Porosidad $(\varepsilon)$ : Los resultados obtenidos para los varietales de Ecuador fueron los siguientes (Tabla 2): cacao Nacional con $0.64 \pm 0.04$ y para CCN 51 con $0.54 \pm 0.01$. Para los varietales de Perú, CCN 51 tuvo $0.64 \pm 0.01$ y para ICS 6 con $0.64 \pm 0.01$. El grano de cacao CCN 51 de Ecuador se reportó como diferente entre los demás granos de cacao, y el 18.51 \% menos poroso que los demás cultivares de cacao, que no existe diferencia significativa ( $p>0.05$ ) entre ellos. La porosidad del grano de cacao CCN 51 está relacionada directamente con el índice de fermentación con un $r^{2} 83.34$ \% (Rivera, 2018), el cultivar CCN 51 de Ecuador es el grano de cacao con menor porosidad, debido a que su índice de fermentación (IF) fue menor, de ahí la importancia de controlar la porosidad del grano de cacao para producir chocolate de calidad.

Índice de fermentación (IF): Los valores IF obtenidos para los varietales de Ecuador fueron los siguientes: el cacao Nacional llegó a $77.66 \pm 1.33 \%$, con 72 horas de fermentación; y el cacao CCN 51 alcanzó un $58.86 \pm 1.45$ \%, con 144 horas de fermentación. Este bajo nivel de fermentación se debe a que en los días de fermentación y secado hubo lluvias constantes, y el secado se realizó a la sombra. Los cacaos del Perú mostraron el siguiente IF: CCN 51 con 80.68 $\pm 1.85 \%$, e ICS6 con valor de $74.64 \pm 2.64 \%$. El grano de cacao con mayor nivel de fermentación fue el CCN 51 de Perú, es decir, tiene un buen nivel para la elaboración de chocolates (Jiménez, Amores y Solórzano, 2014), y el menos fermentado fue el CCN 51 de Ecuador. Los granos de cacao ICS 6 y Nacional de Ecuador se consideran con igual nivel de fermentación ( $p>0.05$ ).

Se han obtenido resultados de la fermentación con granos de cacao de Ecuador fino de aroma, que se fermentaron en cajones de madera (FC), en sacos de polipropileno (FSP) y en sacos de yute (FSY). Se evaluó a las 96 horas en FC y se obtuvieron valores de 70 a $90 \%$ de granos de cacao fermentados, y a las 120 horas valores, entre 70 y $80 \%$ en (FSP) y (FSY). El mayor valor de índice de fermentación se observó en los sacos de yute con 80 \% de granos fermentados (Bravo y Mingo, 2011), otros autores reportan valores similares para grano de cacao de Ecuador (Ruiz et al., 2015); estos valores no difieren de los resultados obtenidos. En referencia a la tabla de clasificación de calidad de cacao de Ecuador (INEN, 2006), relacionado al índice de fermentación, el grano de cacao Nacional se encontró en la clasificación ASSS (Arriba Superior Summer Selecto) por alcanzar el $77.66 \pm 1.33 \%$ comparado con el total mínimo fermentado de 75 \%, y según el índice de grano en la clasificación ASS (Arriba Superior Selecto) (120-125) porque obtuvo un valor de $124 \mathrm{~g}$ de peso en 100 granos de cacao. El grano de cacao CCN 51 mostró un valor en índice de grano superior al rango de la tabla, con valor de $150 \mathrm{~g}$ de peso en 100 granos de cacao, y alcanzó un índice de fermentación de $58.86 \pm 1.45 \%$, valor inferior a lo establecido de 76 \% (INEN, 2006). En Perú (Tingo María) se han obtenido resultados de fermentación de cacao usando transferencia manual y semimecanizada durante la fermentación con resultados 
de índice de fermentación de 87.30 \% y 91.67 \% respectivamente (Peláez, Guerra y Contreras, 2016). En otra investigación realizada en la región de San Martín (Uchiza) con el cultivar CCN 51 se obtuvo un porcentaje de fermentación de 89.70 (Loo, 2019).

Índice de blancura (IB): En la Tabla 2, se muestran los siguientes resultados para los cultivares de Ecuador: el cacao Nacional con $31.40 \pm 0.22$, y para el CCN 51 con un valor de 31.40 \pm 0.25 ; no muestran diferencias significativas ( $p>0.05$ ) entre ellos. Los materiales de Perú, ICS 6 con $25.87 \pm 0.32$ y CCN51 con $28.93 \pm 0.38$, muestran diferencias significativas ( $\leq \leq 0.05$ ), además, son más oscuros por tener valores menores de IB que los materiales de Ecuador. En el proceso tecnológico los granos de cacao pasan por operaciones unitarias que contribuyen a incrementar el color oscuro (reacción de maillard), tales como tostado y concado, por lo tanto, se estima que los granos de cacao bajen sus valores de IB en el procesamiento. Otros autores (Chire, Orihuela y Ureña, 2017) reportan valores de IB para chocolates oscuros comercializados en el Perú en el rango de $27.12 \pm 0.20$ a $29.72 \pm 0.26$. Estos no son un limitante en la calidad del chocolate que elabore la industria ni para el cliente que lo consume.

Tabla 2. Valores físicos de los cultivares de cacao

\begin{tabular}{|c|c|c|c|c|c|}
\hline \multirow{2}{*}{$\begin{array}{c}\text { Tratamientos } \\
\text { de cacao }\end{array}$} & \multicolumn{5}{|c|}{ Variables } \\
\hline & $I G(g)^{*}$ & Testa (\%)* & $\varepsilon(\%)^{*}$ & IF $(\%)^{*}$ & $\mathrm{IB}^{*}$ \\
\hline Nacional Ecuador & $1.24 \pm 0.03^{d}$ & $16.19 \pm 0.49^{a}$ & $0.64 \pm 0.04^{a}$ & $77.66 \pm 1.33^{\mathrm{ab}}$ & $31.40 \pm 0.22^{\mathrm{a}}$ \\
\hline CCN 51 Ecuador & $1.50 \pm 0.02^{a}$ & $12.33 \pm 0.34^{b}$ & $0.54 \pm 0.01^{b}$ & $58.86 \pm 1.45^{c}$ & $31.40 \pm 0.25^{\mathrm{a}}$ \\
\hline CCN 51 Perú & $1.41 \pm 0.06^{b}$ & $10.05 \pm 0.54^{c}$ & $0.64 \pm 0.01^{\mathrm{a}}$ & $80.68 \pm 1.85^{a}$ & $28.93 \pm 0.38^{b}$ \\
\hline ICS 6 Perú & $1.30 \pm 0.01^{c}$ & $15.38 \pm 0.37^{a}$ & $0.64 \pm 0.01^{a}$ & $74.64 \pm 2.64^{b}$ & $25.87 \pm 0.32^{c}$ \\
\hline
\end{tabular}

*La desviación estándar seguida de letras distintas en la misma columna es significativamente diferente ( $p \leq 0.05)$.

Acidez: En la Tabla 3, se muestran los valores para la acidez titulable. Existe diferencia significativa $(p \leq 0.05)$ entre la media de acidez para todos los tipos de granos de cacao, y los valores de acidez para los materiales de Perú, que son tres veces mayores a los valores de acidez para los materiales de Ecuador; esta diferencia puede ser debido al origen y a la poscosecha. Existe una diferencia de valores de acidez a los reportados en los granos de cacao en el estado de Miranda (Venezuela) que alcanzó 1.22 \% de acidez, este valor se ubica entre los valores de acidez de Ecuador y Perú (Lares, Gutiérrez, Pérez y Álvarez, 2012). En el concado (conchado), operación unitaria que desarrolla los sabores del chocolate, la acidez cambia durante las horas de conchado de $1.60 \%$ a 0.60-0.80 \%, se volatiliza $30 \%$ aproximadamente de ácido acético y hasta 50 \% de aldehídos de bajo punto de ebullición (Ley citado en Beckett, 1994).

Tabla 3. Valores de acidez y pH de los cultivares de cacao

\begin{tabular}{|l|l|l|}
\hline \multicolumn{1}{|c|}{ Tratamientos de cacao } & \multicolumn{1}{|c|}{ Promedio acidez $(\%)^{*}$} & \multicolumn{1}{c|}{${\text { Promedio } \mathrm{pH}^{*}}^{*}$} \\
\hline Nacional Ecuador & $0.82 \pm 0.03^{\mathrm{c}}$ & $5.18 \pm 0.04^{\mathrm{b}}$ \\
\hline CCN 51 Ecuador & $0.61 \pm 0.03^{\mathrm{d}}$ & $5.36 \pm 0.01^{\mathrm{a}}$ \\
\hline CCN 51 Perú & $2.05 \pm 0.09^{\mathrm{b}}$ & $5.15 \pm 0.06^{\mathrm{b}}$ \\
\hline ICS 6 Perú & $2.49 \pm 0.05^{\mathrm{a}}$ & $4.93 \pm 0.07^{\mathrm{c}}$ \\
\hline
\end{tabular}

*La desviación estándar seguida de letras distintas en la misma columna es significativamente diferente ( $p \leq 0.05)$. 
pH: Como se observa en la Tabla 3, existen diferencias significativas $(p \leq 0.05)$ entre los valores de $\mathrm{pH}$ de los varietales en estudio, salvo el caso de los granos de cacao Nacional de Ecuador y CCN 51 de Perú, estos valores no difieren mucho de los de la investigación hecha en Maracaibo (Venezuela), que reporta valores de pH para el grano de cacao porcelana de 5.12 y 5.23, a los tres y cuatro días de fermentación respectivamente (Portillo, Graziani de Fariñas y Betancourt, 2007). En otra investigación llevada a cabo en Brasil, se reportan valores en un rango de 5.2 a 6.3 (Loureiro et al., 2017). Se observa también que el grano de cacao ICS 6 tiene el menor valor de $\mathrm{pH}$, lo que corresponde a una alta acidez. En el concado (conchado), los valores de $\mathrm{pH}$, cambiarán cuando se tiene el chocolate como producto terminado.

Análisis proximal: Los resultados del análisis proximal se muestran en la Tabla 4. Para la humedad en el grano de cacao no muestra diferencias significativas $(p \leq 0.05)$ entre los granos de cacao de Ecuador y CCN 51 de Perú; sin embargo, es diferente en humedad el grano de cacao ICS 6 de Perú, posiblemente debido a que el secado solar ha sido mayor. Estos valores de humedad para los granos de cacao de Ecuador y Perú son superiores a los reportados en un trabajo en el estado de Miranda (Venezuela) para el grano de cacao fermentado y seco, que reportó valores de $4.31 \pm 0.06 \%$ de humedad (Lares et al., 2012). Otro estudio desarrollado en Mérida (Venezuela) reporta valores de humedad del grano de cacao entre 7.5 a 7.7 \% (Zambrano, Gómez, Ramos, Romero, La Cruz, et al, 2010). La humedad es importante porque valores superiores al 7 $\%$ pueden causar problemas con hongos y desmejorar la calidad del grano almacenado.

Las proteínas muestran diferencias significativas ( $\mathrm{p} \leq 0.05$ ), siendo las proteínas del grano de cacao ICS 6 la mayor con $15.58 \pm 0.28 \%$, seguida de CCN 51 del Perú con $14.23 \pm 0.26 \%$, superiores a los niveles proteicos de los granos de Ecuador. Sin embargo, los valores de proteínas de los cacaos del Perú son similares a los obtenidos en República Dominicana para granos de cacaos forasteros con 15.29 \% y trinitarios con 16.13 \% (Ventura, María, González, Rodríguez y Almonte, 2014).

El contenido de grasa para los granos de cacao de Ecuador no muestra diferencia significativa ( $p>0.05$ ): cacao Nacional con $50.87 \pm 0.19 \%$ y CCN51 con $51.02 \pm 0.24 \%$. No obstante, los granos de cacao para los cultivares de Perú muestran las siguientes diferencias significativas ( $p \leq 0.05$ ): ICS 6 con $45.28 \pm 0.45 \%$ y CCN 51 con $47.28 \pm 0.22 \%$. Estas diferencias pueden deberse a los niveles de fermentación de los granos de cacao; por ejemplo, los granos de cacao de Ecuador presentaron menores niveles de fermentación, por lo que los niveles de grasa fueron mayores; y para el caso de los varietales peruanos, el nivel de fermentación fue mayor, por lo tanto, el contenido de grasa fue menor. Algunas fábricas procesadoras de manteca de cacao buscan comprar granos de cacao no fermentados o con un nivel menor de fermentación, por lo que los granos de cacao de Ecuador procesados en tales condiciones serían preferidos por estas compañías. El valor reportado en una investigación muestra que el grano de cacao de Miranda (Venezuela) obtuvo un $46.27 \pm 0.30 \%$ de grasa (Lares et al., 2012), valor ligeramente menor a los granos de cacao de este estudio. En otro estudio se muestran resultados en contenido de grasa de varios materiales de cacao, en un rango de 51.4 \% a 57.7 \% (Perea et al., 2011), este valor mínimo es muy similar a los materiales de Ecuador. Existen otros valores similares en grasa para cacao fino $\leq 50 \%$, y forasteros $\geq 52 \%$ (Verdesoto, 2009), rango de valores de contenido de grasa en cacaos de México reportan de 26.3 a 46.1 \% (Ramírez-González, Cely-Niño y Ramírez, 2013). Para la industria chocolatera es fundamental adquirir granos de cacao con mayor contenido de grasa, ya que esta no solo contribuye al sabor sino también al transporte de la masa de chocolate a través de las tuberías, bombas y máquinas. 
En el contenido de ceniza existe diferencia significativa ( $p \leq 0.05)$ entre los cultivares, siendo superior el material CCN 51 de Ecuador con $2.73 \pm 0.08 \%$, y el menor para CCN 51 de Perú con $2.22 \pm 0.19 \%$; otra investigación reporta valores de ceniza en cacao, en Mérida (Venezuela), mayores con $2.96 \pm 0.05 \%$ (Lares et al., 2012).

Como indica el método, el resultado de carbohidratos totales se obtiene por la diferencia de la suma de los resultados del análisis proximal restados de 100, y los valores son los siguientes: para el cacao Nacional de Ecuador fue de $32.28 \pm 0.46 \%$, y CCN 51, de un valor de $32.17 \pm$ $0.11 \%$. Asimismo, los materiales de Perú obtuvieron los siguientes valores: ICS 6 con $31.24 \pm$ $0.39 \%$ y CCN 51 con un valor de $30.24 \pm 0.31 \%$.

El contenido de fibra cruda para los granos de cacao de Ecuador: Nacional con $4.64 \pm 0.47$ $\%$ y CCN 51 con $4.28 \pm 0.01 \%$ no muestran diferencias significativas ( $p>0.05$ ); sin embargo, los resultados de fibra cruda para los materiales de Perú sí mostraron diferencias significativas ( $\leq \leq 0.05)$ y fueron las siguientes: CCN 51 de $5.74 \pm 0.16 \%$ e ICS 6 de $7.10 \pm 0.28 \%$, valores superiores a los obtenidos con materiales de Ecuador. En un ensayo realizado en Colombia se muestran resultados con un rango de 1.5 a $4.1 \%$ (Perea et al., 2011), rango de valores que está por debajo de los obtenidos en materiales Ecuador y Perú. Se destaca la presencia elevada de fibra cruda para el varietal ICS 6 de Perú con $7.10 \pm 0.28 \%$.

Finalmente, los resultados de proteína y fibra cruda para el grano de cacao ICS 6 peruano tienen potencial para el diseño de chocolates con valor nutritivo.

Tabla 4. Análisis proximal de los cultivares de cacao

\begin{tabular}{|l|c|c|c|c|c|c|}
\hline \multirow{2}{*}{\begin{tabular}{c}
\multirow{2}{*}{$\begin{array}{c}\text { Trat. De } \\
\text { cacao }\end{array}$} \\
\cline { 2 - 7 }
\end{tabular}} & $\begin{array}{c}\text { Humedad } \\
(\%)^{*}\end{array}$ & $\begin{array}{c}\text { Proteína } \\
\text { cruda }(\%)^{*}\end{array}$ & $\begin{array}{c}\text { Grasa cruda* } \\
(\%)\end{array}$ & $\begin{array}{c}\text { Ceniza } \\
(\%)^{*}\end{array}$ & $\begin{array}{c}\text { Carbohidra- } \\
\text { tos totales } \\
(\%)^{*}\end{array}$ & $\begin{array}{c}\text { Fibra cruda } \\
(\%)^{*}\end{array}$ \\
\hline $\begin{array}{l}\text { Nacional } \\
\text { ECuador }\end{array}$ & $6.03 \pm 0.10^{\mathrm{a}}$ & $8.60 \pm 0.20^{\mathrm{c}}$ & $50.87 \pm 0.19^{\mathrm{a}}$ & $2.23 \pm 0.08^{\mathrm{bc}}$ & $32.28 \pm 0.46^{\mathrm{a}}$ & $4.64 \pm 0.47 \mathrm{c}$ \\
\hline CCN 51 Ecuador & $6.00 \pm 0.09^{\mathrm{a}}$ & $8.08 \pm 0.25^{\mathrm{d}}$ & $51.02 \pm 0.24^{\mathrm{a}}$ & $2.73 \pm 0.08^{\mathrm{a}}$ & $32.17 \pm 0.11 \mathrm{a}$ & $4.28 \pm 0.01 \mathrm{c}$ \\
\hline CCN 51 Perú & $6.02 \pm 0.04^{\mathrm{a}}$ & $14.23 \pm 0.26^{\mathrm{b}}$ & $47.28 \pm 0.22^{\mathrm{b}}$ & $2.22 \pm 0.19^{\mathrm{c}}$ & $30.24 \pm 0.31 \mathrm{c}$ & $5.74 \pm 0.16^{\mathrm{b}}$ \\
\hline ICS 6 Perú & $5.49 \pm 0.07^{\mathrm{b}}$ & $15.58 \pm 0.28^{\mathrm{a}}$ & $45.28 \pm 0.45^{\mathrm{c}}$ & $2.41 \pm 0.09^{\mathrm{b}}$ & $31.24 \pm 0.39^{\mathrm{b}}$ & $7.10 \pm 0.28^{\mathrm{a}}$ \\
\hline
\end{tabular}

*La desviación estándar seguida de letras distintas en la misma columna es significativamente diferente ( $p \leq 0.05)$.

\section{Conclusiones y recomendaciones}

Los cultivares Nacional y CCN 51 del Ecuador e ICS 6 y CCN 51 de Perú presentaron entre ellos diferencias y similitudes en su caracterización física y química, dada por la evaluación de las dimensiones de los granos, índice de grano, porcentaje de testa, índice de fermentación, porosidad, índice de blancura, acidez, pH y el análisis proximal.

Por las dimensiones y el indice de grano, los cultivares ecuatorianos al ser menores que los peruanos requerirán temperaturas y tiempos de tostado menores a los peruanos. Al tener mayores valores de porcentaje de testa los cultivares Nacional de Ecuador y el ICS 6 de Perú tendrán menor rendimiento para obtener manteca de cacao que los otros dos cultivares estudiados. 
El índice de fermentación y la porosidad del grano encontrado en el CCN 51 del Ecuador, tuvieron valores inferiores a los otros tres cultivares estudiados, se concluye que por condiciones bioclimáticas no se desarrolló un adecuado proceso de fermentado, lo que menguará la calidad sensorial de los chocolates elaborados a partir de este cultivar. Se recomienda usar cajas fermentadoras cuando prevalecen condiciones húmedas y de temperaturas bajas para controlar los agentes externos que pueden modificar la calidad del grano resultante.

El índice de blancura en los cultivares ecuatorianos estudiados es más homogéneo que el de los peruanos, lo que varía la pigmentación clara y oscura con la diversidad de regiones cacaoteras.

Asimismo, se debe resaltar la diferencia en valores de acidez y pH entre cultivares estudiados, lo que permite abarcar distintos gustos y variadas presentaciones de los chocolates obtenidos a partir de ellos. El valor de humedad del ICS 6 fue menor que el de los demás cultivares estudiados, por lo que se recomienda un manejo adecuado para evitar sobrepasar valores del 7 \% y así prevenir la presencia de hongos que desmejoran la calidad del grano.

Los niveles de grasa y fibra cruda de los cultivares de Ecuador no muestran diferencias entre ellos, pero al compararlos con los del Perú muestran un incremento aproximado de $10 \%$ de grasa, lo que hace que los granos de cacao ecuatorianos sean potencialmente preferidos para los procesadores de manteca de cacao. Sin embargo, con respecto al contenido de fibra cruda en el grano de cacao ICS 6 peruano, presenta alto contenido de esta dentro de sus componentes, lo que beneficia a la alimentación saludable porque la fibra un elemento necesario para el proceso digestivo en los seres humanos.

Las propiedades de cenizas y carbohidratos totales fueron diferentes entre los cultivares de Ecuador y Perú, los granos de cacao del Perú mostraron un incremento en $44 \%$ de proteínas más que los granos de Ecuador, aspecto que prefieren las empresas que elaboran mezclas alimenticias, por lo que estas propiedades químicas influyen en la calidad nutricional y marcan la diferencia para elaborar chocolates con altos niveles proteínico.

Por lo expuesto, el cacao Nacional de Ecuador y el ICS 6 de Perú resultan ser los más competitivos para un mercado cada vez más exigente, por lo tanto, se recomienda ejecutar nuevas investigaciones para su aprovechamiento industrial.

\section{Bibliografía}

Aguilar, H. (2016). Manual para la evaluación de la calidad del grano de cacao. La Lima, Honduras, Editorial FHIA. 22 p.

Alegría, E. (2015). Evaluación de tratamientos previos al proceso de tostado de semillas de cacao para el diseño del área de producción de pasta de cacao (Theobroma cacao) (tesis de ingeniería). Escuela Politécnica Nacional, Ecuador.

Álvarez, C.; Pérez, E, y Lares, M. (2007). Caracterización física y química de almendras de cacao fermentadas, secas y tostadas cultivadas en la región de Cuyagua, estado Aragua. Agronomía Tropical, 57(4), 249-256.

Álvarez, C.; Tovar, L.; García, H.; Morillo, F.; Sánchez, P.; Girón, C., y De Farías, A. (2010). Evaluación de la calidad comercial del grano de cacao (Theobroma cacao L.) usando dos tipos de fermentadores. UDO Agrícola 10 (1): 76-87.

Alianza Cacao Perú. (2016). Foro: Desarrollo Alternativo: Intercambio de Experiencia Perú - Colombia. Recuperado el 15 de noviembre de 2018 de https://www.unodc.org/documents/peruandecuador/DocumentosDA/PeruColombiaDA/1._PRESENTACION_ALIANZA_CACAO_PERU_NNUU. 
Amores, F.; Palacios, A.; Jiménez, J., y Zhang, D. (2009). Entorno ambiental, genética, atributos de calidad y singularización del cacao en el nororiente de la provincia de Esmeraldas. INIAP-SENACYTAPROCANE-USDA. INIAP, Estación Experimental Tropical Pichilingue. (Quevedo, EC). Boletín técnico (135), 5-99.

Asociación Nacional de Exportadores de Cacao (ANECACAO). (2019). Cacao CCN 51. Recuperado el 15 de agosto de 2019 de http://www.anecacao.com/es/quienes-somos/cacaoccn51.html

Association of Official Analytical Chemists (AOAC). (2016). Official Methods of Analysis of Association of AOAC International. $18^{\text {th }}$ Edition, Volume II. Editors: W. Horwitz and G. W. Latimer, Jr. Maryland, USA.

Asociación Peruana de Productores de Cacao (APPCACAO). (2012). Manual de control de calidad del cacao. Lima, Perú.

Bravo, N., y Mingo, F. (2011). Valoración de tres métodos de fermentación y secado para mejorar la calidad y rentabilidad del cacao fino de aroma (Theobroma cacao L.) en la parroquia Panguintza del cantón Centinela del Cóndor, provincia de Zamora Chinchipe (tesis de ingeniería). Universidad de Loja, Ecuador.

Caballero, J.; Avendaño, C.; González, N., y López, S. (2016). Influencia del tipo de cacao (Theobroma cacao L.) en las características del fermentado y secado. Agroproductividad, 9(1), 48-54.

Chire, G.; Valdivia, R.; Orihuela, C., y Ureña, M. (2017). Assessment of physical and physicochemical quality of main chocolates traded in Peru. Acta Agronómica, 66(2), 164-171.

Chire, G.; Verona, P., y Guzmán, J. (2016). Cambios en el color durante el beneficio del grano de cacao (Theobroma cacao L.) peruano procedente de Piura. Ciencia e Investigación, 19(1), 29-34.

Chire, G.; Orosco, P.; Valdivia, R., y Ureña, M. (2014). Determinación de las propiedades físicas, químicas y termofísicas de diferentes granos de cacao del Perú. CIBIA IX, 3(9), 545-551.

Food and Agriculture Organization - Instituto Interamericano de Cooperación para la Agricultura (FAO IICA). (2007). Estudio de caso: denominación de origen cacao arriba.

Revalorización del cacao arriba. Recuperado de http://orton.catie.ac.cr/REPDOC/A7704E/A7704E.PDF. 70 p.

Federación Nacional de Cacaoteros (FEDECACAO). (2004). El beneficio y características físico-químicas del cacao (Theobroma cacao L.). Bogotá, Colombia: Editorial Produmedios.

Gutiérrez, M. (2007). Manual: Prácticas de control de calidad de cacao en centro de acopio.

Gobierno Regional de Piura. Programa de Desarrollo Rural Sostenible de la GTZ. Recuperado de https:// censalud.ues.edu.sv/CDOC-Deployment/documentos/Pr_cticas_de_control_de_calidad_de_cacao.pdf

Instituto Nacional de Calidad (INACAL). (2016). NTP - ISO 1114:2016 Granos de cacao. Prueba de Corte. Lima, Perú.

Instituto Ecuatoriano de Normalización (INEN). (2006). NTE 176:2006. Cacao en granos. Requisitos. Cuarta revisión.

Jiménez, J.; Amores, F., y Solórzano, E. (2014). Componentes de identidad para reconocer las diferencias del cacao que se produce en varias regiones del Ecuador. Estación Experimental Tropical Pichilingue. INIAP, Boletín Técnico: 164. Recuperado el 25 de abril de 2018 de http://repositorio.iniap. gob.ec/bitstream/41000/3561/1/iniapeetp-BT-164.pdf

Lares, M.; Gutiérrez, R.; Pérez, E., y Álvarez, C. (2012). Efecto del tostado sobre las propiedades físicas, fisicoquímicas, composición proximal y perfil de ácidos grasos de la manteca de granos de cacao del estado Miranda, Venezuela. Revista Científica UDO Agrícola, 12(2), 439-446.

Ley, D. (1994). Conchado. En Beckett, S.T. (eds.). Fabricación y utilización industrial del chocolate. Zaragoza, España: Editorial Acribia.

Lohman, MH., y Hartel, RW. (1994). Effect of milk fat fractions on fat bloom in dark chocolates. Journal of American Oil Chemists' Society, 71(3), 267-276. 
Loo, J. (2019). Variación de la conductividad eléctrica del medio acuoso por inmersión de granos de cacao CCN-51 con diferentes índices de fermentación (tesis de ingeniería), Universidad Nacional Agraria La Molina, Perú.

Loureiro, G.; Reis de Araujo, Q.; Valle, R.; Andrade, G., y Moreira de Souza, S. (2017). Influencia de factores agroambientales sobre la calidad del clon de cacao (Theobroma cacao L.) PH-16 en la región cacaotera de Bahía, Brasil. Ecosistemas y Recursos Agropecuarios, 4(12), 579-587.

MINAGRI-DGPA-DEEIA. (2016). Estudio del cacao en el Perú y en el Mundo: Un análisis de la producción y el comercio. Recuperado de file:///C:/Users/TOSHIBA/Downloads/estudio-cacao-peru-julio-2016\%20(4).pdf

Nogales, J.; Graziani, L., y Ortiz, L. (2006). Cambios físicos y químicos durante el secado al sol del grano de cacao fermentado en dos diseños de cajones de madera. Agronomía Tropical, 56(1), 5-20.

Ortiz, L.; Graziani, L., y Rovedas, G. (2009). Influencia de varios factores sobre características del grano de cacao fermentado y secado al sol. Agronomía Tropical, 59(2), 119-27.

Peláez, P., Guerra, S., y Contreras, D. (2016). Changes in physical and chemical characteristics of fermented cocoa (Theobroma cacao) beans with manual and semi-mechanized transfer, between fermentation boxes. Scientia Agropecuaria, 7(2), 111-119.

Perea, J.; Ramírez, O., y Villamizar, A. (2011). Caracterización fisicoquímica de materiales regionales de cacao colombiano. Biotecnología en el Sector Agropecuario y Agroindustrial, 9(1), 35-42.

Pérez, E.; Álvarez, C., y Lares, M. (2002). Caracterización física y química de almendras de cacao fermentado, seco y tostado de la región de Chuao. Agronomía Tropical, 52(2), 161-172.

Portillo, E.; Graziani de Fariñas, L., y Betancourt E. (2007). Análisis Químico del Cacao Criollo Porcelana (Theobroma cacao L.) en el Sur del Lago de Maracaibo. Facultad de Agronomía de la Universidad del Zulia, 24 (3), 522-546.

Ramírez-González, MB.; Cely-Niño, VH., y Ramírez, SI. (2013). Actividad antioxidante de clones de cacao (Theobroma cacao L.) finos y aromáticos cultivados en el estado de Chiapas-México. Perspectivas en Nutrición Humana, 15(1), 27-40.

Rivera, J. (2018). Correlación de la porosidad con el grado de fermentación del grano de cacao peruano (Theobroma cacao L.) (tesis de ingeniería), Universidad Nacional Agraria La Molina, Perú.

Rivera, R.; Mecías, F.; Guzmán, A.; Peña, M.; Medina, H.; Casanova, L.; Barrera, A., y Nivela, P. (2012). Efecto del tipo y tiempo de fermentación en la calidad física y química del cacao (Theobroma cacao L.) tipo nacional. Ciencia y Tecnología, 5(1), 7-12.

Ruiz, M.; Mera, O.; Prado, A., y Cedeño, W. (2015). Influencia de la época de cosecha en la calidad del licor de cacao tipo nacional. ESPAMCIENCIA, 5(2), 73-85.

Ureña, M. (1990). Obtención de alimentos modelo a partir de bentonita, glicerol y agua, para estudios de transferencia de calor (tesis de doctorado). Universidad Politécnica de Valencia, España.

Vargas, P.; Ciobota, V.; Salinas, W.; Kampe, B.; Aponte, PM.; Rosch, P.; Popp, J., y Ramos, LA. (2016). Distinction of Ecuadorian varieties of fermented cocoa beans using Raman spectroscopy. Food Chemistry, 211, 274-280.

Ventura, M.; María, A.; González, J.; Rodríguez, O., y Almonte, J. (2014). Caracterización de los atributos de calidad del cacao (Theobroma cacao L.) del municipio de Castillo. Revista Agropecuaria y Forestal APF, 3(1), 55-60.

Vera, J.; Vallejo, Ch.; Párraga, D.; Morales, W.; Macías, J., y Ramos, R. (2014). Atributos físicos-químicos y sensoriales de las Almendras de quince clones de cacao nacional (Theobroma cacao L.) en el Ecuador. Ciencia y Tecnología, 7(2), 21-34.

Verdesoto, P. (2009). Caracterización química preliminar de cacao (Theobroma cacao) de los municipios de Omoa y La Masica, Honduras (tesis de ingeniería). Universidad Zamorano, Honduras.

Zambrano, A.; Gómez, A.; Ramos, G.; Romero, C.; La Cruz, C., y Rivas, E. (2010). Caracterización de parámetros físicos de calidad en almendras de cacao criollo, trinitario y forastero durante del proceso de secado. Agronomía Tropical, 60(4), 389-396. 